\title{
VARIABLE CURVATURE PHANTOM
}

Ivan Rosenberg, Miguel Awschalom and Randall ir. Ten Haken

\section{Purpose}

The variable cuvature dosimetry phantom described in this note was developed to test the accuracy of the dose modification algorithms used in the treatment planning program developed at the Fermilab Neutron Therapy Facility to estimate dose distributions inside patient contours.

The basic beam model used in the Fermilab program consists of a series of functional algorithms for Central Axis Depth Dose (CADD), depending on Equivalent Square at the surface (ESQ) only, and off-axis ratios (OAR), depending on the field width and the depth in tissue. These algorithms were fitted to a set of dose distributions measured in a large dosimetry phantom for several beams incident normally onto a flat surface.

As patient contours and beam placements in clinical practice seldom reproduce these simple conditions, the dose distribution inside a curved contour for beams at different incident angles has to be modified to take account of these factors. Several 
different approaches can be chosen to accomplish this aim, but all have to address two basic questions: first, for a curved surface, either concave or convex, how does the CADD differ from that appropriate for normal incidence on a flat surface, and, second, how is the OAR affected by the change in tissue depth overlying the calculation points along a transverse plane normal to the beam axis?

To answer the above questions precisely, either a Monte Carlo or a primary/scattered radiation summation approach would be needed. In the case of a functional algorithm model, however, only approximations can be used, based on determining an effective ESQ for the CADD and an effective depth at each off-axis point to calculate the OAR. The only way to test whether these approximations are valid is to compare them against actual measurements under several conditions as far removed from normal incidence on a flat surface as possible. To this end, it was decided to design and build a variable curvature phantom to carry out the measurements.

\section{Design}

The phantom consists of a curved Lucite surface about $40 \mathrm{~cm}$ high bounded by two plates of square cross-section of $45 \mathrm{~cm}$ wide. The curved surface was constructed using arcs of circular cross-sections with 5,10 and $20 \mathrm{~cm}$ radil, either abutting each 
other or joined by straight sections. Three convex curvatures and one concave surface are provided. (Eigure 1)

The top and bottom plates are inscribed with the locations of the centers of curvature of each surface, as well as with the edges of the straight sections. These marks will allow the phantom to be located at known positions relative to the isocenter and, combined with the rotation capabilities of the patient support platform, will permit irradiation with beams at any angle relative to the surfaces.

The size of the phantom was designed to fit the existing two-dimensional remote positioner, so that several points can be sampled with ease. A rim $1 \mathrm{~cm}$ wide has been incorporated in the top opening of the phantom to protect the sensitive volume of the probe ionization chamber from accidental collision with the walls of the phantom during remote positioning. A drainage hole and valve have also been incorporated in the bottom plate to simplify removal of the phantom liquid. 
$T M-1310$

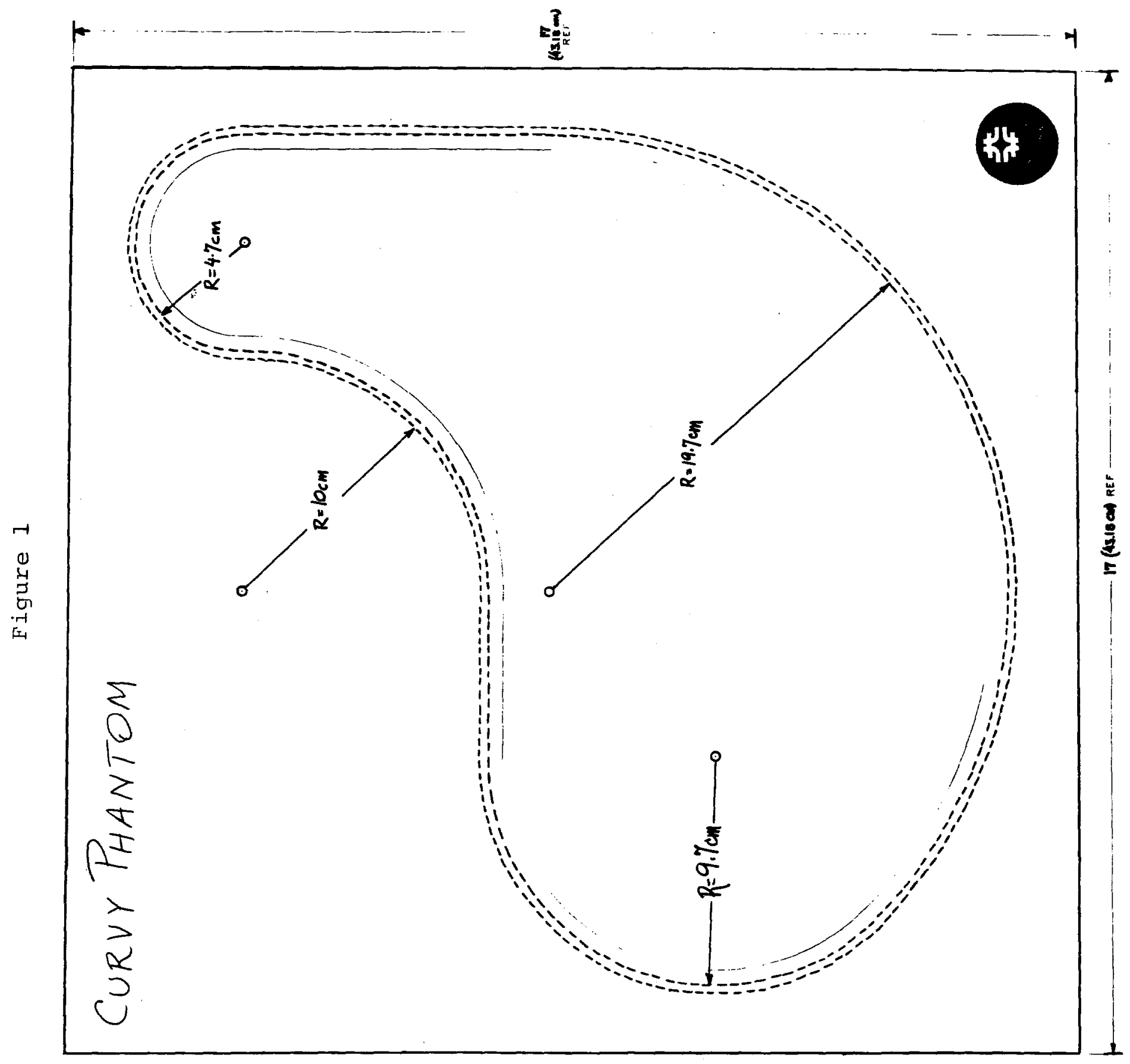

